Cita: Oliveira, H. Z.; Dias, T. S.; Sabino, B.; Dias, C.; Corte-Real, N.; Maia, J. A.; Fonseca, A. (2019). Adaptação transcultural do Empowering Disempowering Motivational Climate Questionnaire para a língua portuguesa e análise da invariância em atletas masculinos brasileiros e portugueses. Cuadernos de Psicología del Deporte, Vol 19(1), 1-18

\title{
Adaptação transcultural do Empowering Disempowering Motivational Climate Questionnaire para a língua portuguesa e análise da invariância em atletas masculinos brasileiros e portugueses
}

\section{Adaptación transcultural del Empowering Disempowering Motivational Climate Questionnaire para el portugués y el análisis de la invarianza en los atletas masculinos brasileños y portugués}

\section{Cross-cultural adaptation of Empowering Disempowering Motivational Climate Questionnaire for Portuguese language and analysis of invariance in Brazilian and Portuguese male athletes}

\author{
Oliveira, Helder Zimmermann ${ }^{1}$, Dias, Teresa Silva ${ }^{1,2}$, Sabino, Bebiana ${ }^{1}$, Dias, Cláudia ${ }^{1}$, Corte-Real, \\ Nuno ${ }^{1}$, Maia, José António ${ }^{1}$, Fonseca, António ${ }^{1}$ \\ ${ }^{1}$ CIFI2D, Faculdade de Desporto, Universidade do Porto $;^{2}$ CIEE, Faculdade de Psicologia e Ciências \\ da Educação, Universidade do Porto.
}

Financiamento:

CAPES - concessão de bolsa para Helder Zimmermann Oliveira (99999.002183 / 2015-03);

FCT - concessão de bolsa para Teresa Silva Dias (UID/CED/ 00167/2013).

\section{RESUMO}

O objetivo do presente estudo foi proceder à tradução e adaptação transcultural do "Empowering and Disempowering Motivational Climate Questionnaire-Coach" (EDMCQ-C) para a língua portuguesa e analisar a invariância da sua estrutura fatorial, quando aplicado a atletas brasileiros e portugueses. A amostra foi constituída por 963 atletas do sexo masculino, praticantes de diferentes desportos (andebol, basquetebol, futebol, futsal e voleibol), com idades compreendidas entre os 11 e os 24 anos $(M=15.04 \pm 1.88)$, sendo 553 brasileiros $(M=$ $15.81 \pm 1.91)$ e 410 portugueses $(M=13.94 \pm 1.30)$. Através do recurso à Análise Fatorial Confirmatória foi possível verificar índices de ajustamento aceitáveis ao modelo teórico examinado tanto para portugueses $\left(\chi^{2} / \mathrm{df}=\right.$ 1.67; $\mathrm{CFI}=0.92 ; \mathrm{TLI}=0.91 ; \mathrm{SRMR}=0.06 ; \mathrm{RMSEA}=0.04)$ quanto para brasileiros $\left(\chi^{2} / \mathrm{df}=1.92 ; \mathrm{CFI}=0.90\right.$; $\mathrm{TLI}=0.89 ;$ SRMR $=0.05 ;$ RMSEA $=0.04)$. Os resultados também evidenciaram que o modelo de medida é invariante entre atletas brasileiros e portugueses $(\triangle \mathrm{CFI}<0.01)$. Em conformidade, os resultados encontrados suportam a sugestão de que a versão adaptada para a língua portuguesa do EDMCQ-C evidencia propriedades psicométricas aceitáveis, podendo, portanto, constituir-se como uma ferramenta útil para avaliar as perceções de atletas brasileiros e portugueses sobre o clima motivacional que lhes é proporcionado pelos seus treinadores.

Palavras chave: Avaliação do clima motivacional, EDMCQ-C, adaptação transcultural, análise psicométrica. 
Oliveira, H. Z.; Dias, T. S.; Sabino, B.; Dias, C.; Corte-Real, N.; Maia, J. A.; Fonseca, A.

\section{RESUMEN}

El objetivo de este estudio fue la adaptación transcultural de la versión en portugués del "Empowering and Disempowering Motivational Climate Questionnaire-Coach" (EDMCQ-C), que evalúa la percepción de los atletas en el clima motivacional proporcionada por el entrenador, e investigar la invariancia factorial entre brasileños y portugueses. La muestra consistía en 963 atletas hombres, practicantes de diferentes deportes (balonmano, baloncesto, fútbol, fustal y voleibol) con edades entre 11 y 24 años $(M=14,15 \pm 1.88)$, de los cuales 553 eran brasileños $(M=15,81 \pm 1,91)$ y 410 atletas portugueses $(M=13,94 \pm 1,30)$. Mediante el análisis factorial confirmatorio fue posible verificar índices de ajuste aceptables al modelo teórico tanto para portugueses $(\chi 2 / \mathrm{df}=$ $1,67, \mathrm{CFI}=0,92, \mathrm{TLI}=0,91$; SRMR $=0.06$; RMSEA $=0: 04)$ cuanto para brasileños $(\chi 2 / \mathrm{df}=1.92, \mathrm{CFI}=0.90$, $\mathrm{TLI}=0.89, \mathrm{SRMR}=0.05, \mathrm{RMSEA}=0.04)$. Los resultados también mostraron que el modelo de medición es invariante entre atletas portuguesas y brasileñas $(\triangle \mathrm{CFI}<0,01)$. En conclusión, el instrumento puede ser una herramienta útil para analizar la percepción de los atletas brasileños y portugueses al clima motivacional proporcionado por el entrenador.

Palabras clave: Evaluación del clima motivacional, EDMCQ-C, adaptación transcultural, análisis psicométrico.

\section{ABSTRACT}

The aim of the present study was to carry out the cross-cultural adaptation of the Portuguese version of "Empowering and Disempowering Motivational Climate" (EDMCQ-C), which evaluates the athletes' perception the motivational climate provided by the coach and to examine the factorial invariance between Brazilians and Portuguese. The sample was composed by 963 male athletes from different sports (handball, basketball, soccer, futsal and volleyball), aged between 11 and $24(\mathrm{M}=15.04 \pm 1.88)$, of whom 553 were Brazilian $(\mathrm{M}=15.81 \pm$ $1.91)$ and 410 Portuguese athletes $(M=13.94 \pm 1.30)$. The results of the confirmatory factorial analysis found satisfactory adjustment indices of the model for both portuguese $(\chi 2 / \mathrm{df}=1.67$; CFI $=0.92 ; \mathrm{TLI}=0.91$; SRMR $=$ 0.06; $\mathrm{RMSEA}=0.04)$ and brazilian $(\chi 2 / \mathrm{df}=1.92 ; \mathrm{CFI}=0.90 ; \mathrm{TLI}=0.89 ; \mathrm{SRMR}=0.05 ; \mathrm{RMSEA}=0.04)$. The results also showed that the model of measurement was invariant between Brazilian and Portuguese athletes $(\Delta C F I$ $<0.01$ ). In conclusion, the instrument used can be a useful to analyze the perception of Brazilian and Portuguese athletes for the motivational climate provided by the coach.

Keywords: Motivational climate analysis, EDMCQ-C, Cross-cultural adaptation, psychometric Analysis.

\section{INTRODUÇÃO}

Nos últimos anos, algumas pesquisas têm procurado compreender os aspectos da relação entre treinador e atleta que influenciam a prática desportiva. A forma como essa relação se estabelece tem sido considerada central na motivação, no desempenho (Mageau \& Vallerand, 2003; Spaccarotella, 2017), e na permanência ou abandono da prática desportiva de jovens atletas (Dias et al., 2018; Lemyre, Roberts, \& Stray-Gundersen, 2007), como também associado a comportamentos antisociais (Leo-Marcos, SanchézMiguel, Sanchéz-Oliva, Gómez-Corrales, \& GarcíaCalvo, 2009) e ao bem estar dos atletas (Castillo, Duda, Álvarez, Mercé, \& Balaguer, 2011) . De entre as diferentes perspectivas sobre a relação treinador/atleta no desporto, duas teorias têm sido destacadas por estudar o impacto do suporte motivacional fornecido pelo treinador na motivação de atletas para a prática desportiva, nomeadamente a
Teoria da Autodeterminação (TAD) (Deci \& Ryan, 1985; Ryan \& Deci, 2017) e a Teoria dos Objetivos de Realização (TOR) (Nicholls, 1984).

Em seu escopo, a TAD caracteriza o comportamento e atitudes do treinador em relação ao ambiente de treino, podendo ser associado ao suporte à autonomia, ao suporte social e/ou um ambiente controlador (Deci \& Ryan, 2000). Em um ambiente de suporte à autonomia, o treinador encoraja os atletas a assumirem a responsabilidade da própria participação no desporto e favorece comportamentos que nutrem os interesses e as preferências dos atletas (Cantú-Berrueto, Castillo, López-Walle, Tristán, \& Balaguer, 2016; Mageau \& Vallerand, 2003; Smith et al., 2016;). De forma oposta, o treinador pode-se comportar de forma coerciva e autoritária, exercendo pressão a fim de impor aos atletas uma maneira específica e pré-concebida de pensar e de se comportar, gerando um ambiente de treino 


\section{Adaptação transcultural do Empowering Disempowering}

controlador (Bartholomew, Ntoumanis, \& Thogersen-Ntoumani, 2009). Neste enquadramento, a TAD também considera o suporte social como fator determinante na motivação dos atletas. Esse suporte acontece quando o treinador respeita os valores de cada um dos atletas, tanto no ambiente desportivo quanto fora dele (Reinboth, Duda, \& Ntoumanis, 2004).

Como consequência desses ambientes, verificou-se que a perceção dos atletas para um ambiente de suporte à autonomia proporcionado pelo treinador promove maior satisfação e diversão em praticar desporto (Ryan \& Deci, 2007). Outras consequências foram apontadas num estudo de revisão, realizado por Occhino, Mallett, Rynne, e Carlisle (2014), onde constataram maiores níveis de motivação, persistência, bem-estar, e menor abandono para os atletas que percebem um ambiente de suporte à autonomia proporcionado pelos treinadores. Em oposição a esse ambiente, a TAD postula que o treinador que promove um ambiente de excessivo controle tem como consequência para os atletas menores níveis de autodeterminação, overtraining e distúrbios alimentares dentre outras respostas maladaptativas (Bartholomew et al., 2009; Pelletier, Fortier, Vallerand, \& Briere, 2001). Simultaneamente, outras investigações com base na TAD afirmam que o suporte social fornecido pelo treinador favorece o desenvolvimento e a satisfação dos atletas, sendo que previne o abandono da prática desportiva (DeFreese \& Smith, 2013; Sheridan, Coffee, \& Lavallee, 2014).

Por outro lado, a TOR propõe a análise do ambiente social e psicológico através do clima motivacional, o qual é caracterizado pela maneira como o treinador encoraja os jogadores a tornarem-se mais ou menos envolvidos para a tarefa ou para o ego (Ames, 1992; Nicholls, 1984). Assim, no contexto desportivo, quando os treinadores desenvolvem uma perceção de competência dos atletas com critérios autorreferenciados, com base no processo de aprendizagem e de melhora pessoal favorecem um maior envolvimento do atleta para a tarefa (Duda \& Balaguer, 2007). Por oposição, quando o treinador favorece um clima interpessoal excessivamente competitivo, no qual a demonstração e a perceção de competência do atleta é marcada por critérios normativos, promoverá um clima de envolvimento ao ego (Duda \& Balaguer, 2007). Associado a isso, num estudo de revisão, Harwood, Keegan, Smith, e Raine, (2015) referem que a perceção dos atletas para climas motivacionais de envolvimento para tarefa tem sido associada a maiores níveis de perceção de competência, autoestima, afetos positivos, atitudes morais, sentimento de flow, entre outros benefícios. Em contrapartida, a perceção de um clima motivacional de envolvimento para o ego é referida como estando associada a maiores níveis de ansiedade, desmotivação, afeto negativo e atitudes antissociais.

Ainda que diversas publicações tragam enorme contributo relativamente a perceção do atleta sobre o ambiente motivacional fornecido pelo treinador e a relação dessa variável em diversos aspetos psicológicos, fazer inferência com base em apenas uma das teorias pode negligenciar aspetos práticos fundamentais (Standage, Duda, \& Ntoumanis, 2005). Neste sentido, Hagger e Chatzisarantis (2007) afirmam que unir teorias sócio cognitivas poderá promover explicações complementares sobre fenômenos comportamentais. Em um estudo pioneiro, Ntoumanis (2001) analisou ligações TAD e a TOR em contextos desportivos, e concluiu que a orientação para a Tarefa prediz motivações mais autodeterminadas e orientação ao ego prediz motivações menos autodeterminadas. Com esse propósito, Duda (2013) propõe um modelo ampliado para o clima motivacional criado pelo treinador com base nessas duas teorias, TOR e TAD, para atletas de desportos coletivos.

Este modelo serve de base para diversas pesquisas (Fenton, Duda, Appleton, \& Barrett, 2016; Smith et al., 2015) e como modelo conceitual de um programa de formação e capacitação de treinadores, cujo objetivo é torná-los aptos a promoverem uma prática desportiva envolvente e prazerosa para os jovens (Castillo, Ramis, Cruz, \& Balaguer, 2013; Duda, 2013; Duda et al., 2013). De acordo com os autores, o clima motivacional pode ser de maior ou menor "empowering" (Empoderamento) quando as características associadas são o envolvimento para tarefa, o suporte à autonomia e o suporte social. Em oposição, o clima motivacional pode ser de "disempowering" (Desempoderamento), quando associado ao envolvimento para o ego e a um treino controlador.

Com base no modelo multidimensional proposto por Duda (2013), Appleton, Ntoumanis, Quested, 
Oliveira, H. Z.; Dias, T. S.; Sabino, B.; Dias, C.; Corte-Real, N.; Maia, J. A.; Fonseca, A.

Viladrich, \& Duda (2016) desenvolveram o questionário "Empowering and Disempowering Motivational Climate Questionnaire-Coach" (EDMCQ-C), que visa avaliar a perceção dos atletas sobre o clima motivacional proporcionado pelo treinador, constituídos por 32 itens agrupados em 5 fatores de primeira ordem: Envolvimento para a Tarefa, Suporte à Autonomia, Suporte Social que formam o fator de segunda ordem Empoderamento; Envolvimento ao Ego e Treino Controlador que constituí o fator Desempoderamento de segunda ordem. No estudo de validação, com uma amostra de 2273 adolescentes de diversas modalidades desportivas coletivas e atletas entre os 9 e 17 anos de idade, Appleton et al. (2016) testaram o EDMCQ-C, de acordo com as indicações teóricas que lhe deram origem e apresentaram uma solução para o modelo de medida hierárquica, multidimensional. Os melhores resultados foram alcançados através do modelo de equações estruturais bifatorial cujo os índices globais de ajustamento em duas diferentes amostras foram: $\chi^{2} / \mathrm{df}=1.68 ; \mathrm{CFI}=0.96 ; \mathrm{TFI}=0.94 ; \mathrm{RMSEA}=0.02 ;$ $\chi^{2} / \mathrm{df}=2.30 ; \mathrm{CFI}=0.96 ; \mathrm{TFI}=0.93 ; \mathrm{RMSEA}=0.03$.

Através da aplicação deste instrumento, foi possível analisar a perceção dos atletas sobre o clima motivacional fornecido pelo treinador de 406 atletas ingleses de diversas modalidades desportivas e ambos os sexos, demonstrando uma participação mais saudável nos praticantes que apresentaram altos índices para a perceção do clima motivacional de empoderamento e baixos níveis de desempoderamento concomitantemente (Appleton \& Duda, 2016). Além disso, Fenton et al. (2016), recorrendo ao EDMCQ-C, verificaram que adolescentes praticantes de desporto que percebem um maior clima de empoderamento possuem uma motivação mais autônoma, maior divertimento e praticam mais atividade física em níveis moderados e intensos.

Para o desenvolvimento da investigação em determinado domínio, são naturalmente necessários instrumentos fiáveis e válidos para proceder à avaliação das diferentes variáveis envolvidas. Nos países lusófonos foram encontradas pesquisas com instrumentos validados para medir o clima motivacional proporcionado pelo treinador apenas no contexto desportivo português. Dessas investigações, nota-se a utilização de dois instrumentos, todos eles com base apenas na TOR, o Perceived Motivational
Climate in Sport Questionnaire (Fonseca \& Biddle, 1995) e o Motivational Climate Sport Youth Scale (Borrego \& Silva, 2012; Monteiro et al., 2018). Por outro lado, no Brasil, como noutros países cujo idioma oficial é o português, não foram encontradas investigações sobre o clima motivacional ou a respeito do ambiente de suporte proporcionado pelo treinador, em grande medida por não haver um instrumento adaptado para esses contextos desportivos.

Quando não existem instrumentos para avaliar as variáveis que se pretende investigar, proceder à adaptação transcultural de instrumentos previamente desenvolvidos noutros contextos parece constituir-se como uma opção mais razoável do que desenvolver novos instrumentos (Burgueño, Granero-Gallegos, Alcaraz-Ibáñez, Sicilia, \& Medina-Casaubón, 2018; Úbeda-Colomer, Pérez-Samaniego, \& Devís-Devís, 2018).

Nesse sentido, o objetivo do presente estudo consistiu em realizar a tradução e adaptação transcultural do "coach-created Empowering and Disempowering Motivational Climate" (EDMCQ-C) para a língua portuguesa, bem como verificar a invariância da sua estrutura fatorial quando aplicado com atletas brasileiros e portugueses, procurando dessa forma contribuir para o incremento do conhecimento sobre a perceção dos atletas face ao clima motivacional que lhes é proporcionado pelos seus treinadores e promover a realização de investigações transculturais sobre este tema envolvendo atletas brasileiros e portugueses.

\section{MÉTODOS}

Para classificar o desenho de investigação utilizou-se o sistema proposto por Ato, López-García, e Benavente (2013) para pesquisas na área de Psicologia. Nesse sentido, o presente estudo enquadrou-se no desenho de investigação instrumental uma vez que pretende analisar as propriedades psicométricas de um instrumento de medida adaptado para o contexto desportivo brasileiro e português.

\section{Amostra}

A amostra foi constituída por 963 atletas do sexo masculino com idades compreendidas entre os $11 \mathrm{e}$ os 24 anos $(M=15.04 \pm 1.88)$, sendo 553 brasileiros com idades entre 11 e os $24(M=15.81 \pm 1.91)$ e 410 


\section{Adaptação transcultural do Empowering Disempowering}

portugueses com idades entre 11 e os 18 anos $(M=$ $13.94 \pm 1.3)$. Os atletas pesquisados eram praticantes regulares de andebol, basquetebol, futebol, futsal e voleibol, com pelo menos um ano de prática na modalidade. Os brasileiros apresentaram tempo de prática compreendido entre 1 e 14 anos $(M=4.49 \pm$ $3.19)$ e portugueses tempo de prática entre 1 e 13 $\operatorname{anos}(M=6.02 \pm 3.07)$.

\section{Instrumentos}

O EDMCQ-C foi desenvolvido e validado originalmente em língua inglesa por Appleton, Ntoumanis, Quested, Viladrich, e Duda (2016). O instrumento é constituído por 32 itens agrupados em 5 fatores de primeira ordem originados de 2 fatores de segunda ordem. A distribuição dos fatores de primeira ordem é a seguinte: Envolvimento para Tarefa (com 9 itens; e.g., "O/A meu/minha treinador/a transmite-nos que todos/as jogadores/as contribuem para o sucesso da equipa/e"), Suporte à Autonomia (5 itens; e.g., "O/A meu/minha treinador/a dá aos jogadores/as escolhas e opções"), Suporte Social (3 itens; e.g., "Posso contar sempre com o/a meu/minha treinador/a, independentemente do que aconteça"), Envolvimento para o Ego (7 itens; e.g., "O/A meu/minha treinador/a pensa que somente os/as melhores/as jogadores/as podem jogar") e Treino Controlador (8 itens; e.g., "O/A meu/minha treinador/a valoriza menos os/as jogadores/as que o desapontam"). Os três primeiros fatores são provenientes do fator de segunda ordem definido por Empoderamento e os dois últimos decorrem do fator Desempoderamento. Os itens são respondidos através do recurso a uma escala do tipo Likert de 5 pontos, na qual 1 corresponde a "discordo totalmente" e 5 corresponde a "concordo totalmente".

\section{Procedimentos de Tradução do instrumento}

A versão original do EDMCQ-C foi traduzida para a língua portuguesa por 3 especialistas bilingues. Posteriormente, os itens traduzidos foram submetidos à apreciação de um júri, constituído por 5 elementos, com domínio em ambas as línguas, especialistas na área de Psicologia do Desporto para determinar a existência de equivalência, em termos semânticos e de conteúdo, entre as versões examinadas. Este procedimento teve por base as sugestões de Vallerand (1989) relativamente à tradução e adaptação transcultural, exceto pelo o procedimento de retrotradução na fase de preparação preliminar. Nesse sentido, o procedimento utilizado foi realizado através das sugestões de Brislin (1980). Outras pesquisas no contexto lusófono utilizaram o mesmo procedimento (Duarte, Garganta, \& Fonseca, 2012; Fonseca \& Brito, 2001; Fonseca \& Fox, 2002). Complementarmente, porém, reconhecendo a existência de ligeiras diferenças entre o modo como alguns termos são redigidos no Brasil e em Portugal, o mesmo júri identificou todas as palavras ou expressões que poderiam estar associadas a diferenças da língua portuguesa falada no Brasil e em Portugal. Em seguida foram acrescentados sinônimos ao lado dessas palavras ou expressões afins, para evitar qualquer dúvida por parte do respondente (e.g., desporto/esporte).

A versão resultante deste processo foi aplicada a 5 atletas brasileiros e a 5 atletas portugueses de diferente sexo, idade e modalidade desportiva praticada, visando identificar eventuais dúvidas ou dificuldades de compreensão, numa primeira aproximação à população alvo de aplicação deste instrumento (Fonseca \& Brito, 2001). Como os inquiridos não evidenciaram quaisquer dificuldades no seu preenchimento, deu-se por concluída esta fase, designando-se a versão final como EDMCQ-Cbp (versão brasileira e portuguesa).

\section{Procedimentos de recolha dos dados}

Inicialmente, o estudo foi apresentado aos clubes e/ou treinadores dos atletas afim de convida-los a participar da pesquisa, com uma breve explicação do objetivo e procedimentos para realização da pesquisa. Quando os representantes dos clubes aceitavam participar da investigação, uma data era marcada para aplicação dos questionários antes ou após uma sessão de treino ou jogo. Em ambos os casos foi lido e assinado um consentimento informado pelos atletas ou responsáveis legais (quando menores de idade), do qual constavam os objetivos da investigação e era salvaguardada a confidencialidade dos dados juntamente com sua única utilização para fins de investigação. Todos os questionários foram aplicados pelos pesquisadores seguindo os mesmos procedimentos nos dois países. Esses procedimentos foram aprovados pela Comissão de Ética da Faculdade de Desporto da Universidade do Porto.

Análise dos dados 
Oliveira, H. Z.; Dias, T. S.; Sabino, B.; Dias, C.; Corte-Real, N.; Maia, J. A.; Fonseca, A.

Inicialmente foram calculadas as estatísticas descritivas que incluíram a média $(M)$, desviopadrão, assimetria $(A s)$ e achatamento $(A c)$. Itens com $A s$ superior a 3 e $A c$ superior a 7 , em valores absolutos, indicam violações à distribuição Normal (Finney \& DiStefano, 2006). Foi calculado, também, o coeficiente alfa de Cronbach $(\alpha)$ sendo aceitável um valor maior ou igual a 0.70 (Kline, 2012). Para verificar a existência de outliers multivariados, utilizou-se a distância de Mahalanobis $\left(\mathrm{D}^{2}\right)$. O valor de significância adotado foi de 0.001, seguindo as recomendações de Hair, Anderson, Tatham e Black (2014).

Para avaliar a validade convergente da escala, foram utilizados testes de validade convergente $\mathrm{e}$ discriminante. A validade convergente foi estimada pela variância extraída média (VEM). De acordo com Hair, Anderson, Tatham e Black (2014) os valores de $\mathrm{VEM}_{\mathrm{j}}$ maiores ou igual a 0.5 indicam validade convergente. A validade discrimante foi analisada e estabelecida quando a VEM para cada fator de primeira ordem foi superior ou igual ao quadrado da correlação entre esses fatores (Byrne, 2010).

Posteriormente, realizou-se a Análise Fatorial Confirmatória (AFC) através da estimação dos parâmetros por máxima verossimilhança. A adequação dos modelos foi avaliada através dos seguintes índices de bondade do ajustamento: rácio do valor do qui-quadrado pelos graus de liberdade $\left(\chi^{2} / \mathrm{df}\right)$ sugeridos por Jöreskog e Sörbom (1989). De acordo com Tabachnick e Fidell (2007) valores abaixo de 2.0 sugerem modelos aceitáveis. Além disso, foi considerado o Comparative Fit Index (CFI) e o Tucker-Lewis index (TLI) cujo valores superiores a 0.90 são considerados adequados (Bentler \& Bonett, 1980). Também foram analisados os valores do Standardized Root Mean Square Residual (SRMR) e Root Mean Square Error of Aproximation (RMSEA), para esses estimadores de ajuste do modelo foram consideradas as sugestões de $\mathrm{Hu}$ e Bentler (1999), onde valores abaixo de 0.08 para o SRMR e 0.06 para o RMSEA indicam uma adequação aceitável do modelo.

Para analisar a invariância do modelo entre os atletas portugueses e brasileiros realizou-se o conjunto de testes sugeridos por Byrne e Stewart (2006) para modelos de segunda ordem. Com o intuito de comparar os modelos com os atletas dos dois países, através da AFC foi necessário definir um conjunto de submodelos de medida aninhados onde diferentes parâmetros foram fixados tornando a equivalência entre os dois países cada vez mais restrita. Dessa forma, o primeiro submodelo definido não apresentou qualquer restrição, e serve de base para as comparações com os submodelos seguintes, portanto é reconhecido como o primeiro passo para a invariância, nomeado como invariância configuracional (Byrne \& Stewart, 2006). A etapa seguinte foi testar a invariância a partir da fixação das cargas fatoriais de primeira e segunda ordem, cuja as análises indicam os valores reconhecidos como invariância de medida. Por fim, foram mantidas as cargas fatoriais das duas ordens fixadas para os pesos fatoriais e fixou-se os interceptos de primeira ordem para em seguida fixar segunda fixar também os interceptos de segunda ordem. Assim as invariâncias ficaram definidas da seguinte forma: i) Invariância configuracional (submodelo sem restrições); ii) Invariância de medida I (as cargas fatoriais de primeira ordem são fixadas); iii) Invariância de medida II (as cargas fatoriais de primeira e segunda ordem são fixadas); iv) Invariância escalar I(as cargas fatoriais de primeira e segunda ordem e os interceptos de primeira ordem são fixados); v) Invariância escalar II (as cargas fatoriais e os interceptos de primeira e segunda ordem são fixados). Para verificar a existência da invariância entre os modelos, adotou-se como valores de referência as diferenças do Confirmatory Fit Index $(\triangle \mathrm{CFI})$, sendo que uma variação inferior ou igual a 0.01 foi considerada como indicadora da equivalência do modelo (Cheung \& Rensvold, 2002). As análises foram realizadas no software IBM SPSS AMOS (v.25, SPSS An IBM Company, Chicago, IL).

\section{RESULTADOS}

Os valores das estatísticas descritivas das amostras separados por nacionalidade estão na tabela 1 . Todos os itens do instrumento apresentaram respostas dentro da escala Likert de 1 a 5 pontos, exceto o item $32 \mathrm{com}$ a amostra de atletas portugueses. No que diz respeito à distribuição univariada das respostas aos itens salientaram um valode $A c=8.22$ no item 32 para a amostra com atletas brasileiros. Em relação aos valores médios e desvios padrões os resultados são similares entre brasileiros e portugueses. Os resultados da distância $\mathrm{D}^{2}$ de Mahalanobis resultados 


\section{Adaptação transcultural do Empowering Disempowering}

mostraram a existência de 7 outliers ( 2 portugueses e 5 brasileiros), que foram eliminados dos cálculos.

Os valores do coeficiente $\alpha$ de Cronbach no Envolvimento para a Tarefa foram $\alpha_{\mathrm{pt}}=, 86$ e $\alpha_{\mathrm{br}}=, 84 \mathrm{e}$ no Envolvimento ao Ego de $\alpha_{\mathrm{pt}}=, 74$ e $\alpha_{\mathrm{br}}=, 72$. Os valores do $\alpha$ nos outros fatores foram menores que 0.70. Ademais os valores foram similares entre portugueses e brasileiros.

Tabela 1.

Estatísticas descritivas dos itens e $\alpha$ de Cronbach por fator

\begin{tabular}{|c|c|c|c|c|c|c|c|c|c|c|c|}
\hline \multicolumn{6}{|c|}{ Portugueses } & \multicolumn{6}{|c|}{ Brasileiros } \\
\hline & Média $\pm \mathrm{DP}$ & Mín & Máx & As & $A c$ & & Média $\pm \mathrm{DP}$ & Mín & $\begin{array}{c}\text { Má } \\
\mathrm{x}\end{array}$ & Sk & $\mathrm{Ku}$ \\
\hline $\operatorname{ET}(\alpha=, 86)$ & & & & & & $\operatorname{ET}(\alpha=0,84)$ & & & & & \\
\hline Item 1 & $4,41 \pm 0,78$ & 1,00 & 5,00 & $-1,35$ & 2,03 & & $4,43 \pm 0,86$ & 1,00 & 5,00 & $-1,47$ & 1,66 \\
\hline Item 4 & $4,48 \pm 0,80$ & 1,00 & 5,00 & $-1,82$ & 3,66 & & $4,50 \pm 0,89$ & 1,00 & 5,00 & $-1,97$ & 3,52 \\
\hline Item 11 & $4,40 \pm 0,84$ & 1,00 & 5,00 & $-1,76$ & 3,82 & & $4,37 \pm 0,91$ & 1,00 & 5,00 & $-1,66$ & 2,72 \\
\hline Item 13 & $4,26 \pm 0,81$ & 1,00 & 5,00 & $-1,20$ & 2,02 & & $4,50 \pm 0,83$ & 1,00 & 5,00 & $-1,93$ & 3,86 \\
\hline Item 17 & $4,29 \pm 0,80$ & 1,00 & 5,00 & $-1,30$ & 2,28 & & $4,33 \pm 0,98$ & 1,00 & 5,00 & $-1,48$ & 1,52 \\
\hline Item 21 & $4,22 \pm 0,89$ & 1,00 & 5,00 & $-1,04$ & 0,74 & & $4,21 \pm 1,05$ & 1,00 & 5,00 & $-1,24$ & 0,73 \\
\hline Item 26 & $4,36 \pm 0,92$ & 1,00 & 5,00 & $-1,56$ & 2,15 & & $4,38 \pm 0,98$ & 1,00 & 5,00 & $-1,61$ & 1,94 \\
\hline Item 28 & $4,27 \pm 0,89$ & 1,00 & 5,00 & $-1,28$ & 1,48 & & $4,32 \pm 0,95$ & 1,00 & 5,00 & $-1,39$ & 1,35 \\
\hline Item 32 & $4,65 \pm 0,62$ & 2,00 & 5,00 & $-1,84$ & 3,24 & & $4,69 \pm 0,73$ & 1,00 & 5,00 & $-2,78$ & 8,22 \\
\hline $\operatorname{SA}(\alpha=0,66)$ & & & & & & $\operatorname{SA}(\alpha=0,55)$ & & & & & \\
\hline Item 3 & $3,77 \pm 1,05$ & 1,00 & 5,00 & $-0,68$ & $-0,11$ & & $3,91 \pm 1,14$ & 1,00 & 5,00 & $-0,78$ & $-0,28$ \\
\hline Item 6 & $4,38 \pm 0,88$ & 1,00 & 5,00 & $-1,64$ & 2,83 & & $4,38 \pm 1,00$ & 1,00 & 5,00 & $-1,67$ & 2,32 \\
\hline Item 15 & $4,17 \pm 0,89$ & 1,00 & 5,00 & $-1,18$ & 1,47 & & $3,94 \pm 1,17$ & 1,00 & 5,00 & $-0,86$ & $-0,24$ \\
\hline Item 20 & $4,32 \pm 0,89$ & 1,00 & 5,00 & $-1,55$ & 2,59 & & $4,28 \pm 1,04$ & 1,00 & 5,00 & $-1,44$ & 1,26 \\
\hline Item 30 & $4,50 \pm 0,76$ & 1,00 & 5,00 & $-1,67$ & 2,84 & & $4,44 \pm 0,93$ & 1,00 & 5,00 & $-1,87$ & 3,32 \\
\hline $\operatorname{SS}(\alpha=0,55)$ & & & & & & $\operatorname{SS}(\alpha=0,51)$ & & & & & \\
\hline Item 8 & $4,32 \pm 0,96$ & 1,00 & 5,00 & $-1,56$ & 2,19 & & $4,32 \pm 1,07$ & 1,00 & 5,00 & $-1,53$ & 1,40 \\
\hline Item 14 & $4,08 \pm 1,16$ & 1,00 & 5,00 & $-1,27$ & 0,83 & & $4,20 \pm 1,15$ & 1,00 & 5,00 & $-1,45$ & 1,22 \\
\hline Item 25 & $3,94 \pm 1,16$ & 1,00 & 5,00 & $-1,00$ & 0,15 & & $3,69 \pm 1,29$ & 1,00 & 5,00 & $-0,61$ & $-0,76$ \\
\hline $\mathrm{EE}(\alpha=0,74)$ & & & & & & $\mathrm{EE}(\alpha=0,72)$ & & & & & \\
\hline Item 5 & $2,43 \pm 1,27$ & 1,00 & 5,00 & 0,44 & $-0,87$ & & $3,41 \pm 1,29$ & 1,00 & 5,00 & $-0,30$ & $-0,89$ \\
\hline Item 9 & $2,32 \pm 1,28$ & 1,00 & 5,00 & 0,65 & $-0,69$ & & $2,86 \pm 1,43$ & 1,00 & 5,00 & 0,15 & $-1,29$ \\
\hline Item 10 & $3,12 \pm 1,26$ & 1,00 & 5,00 & $-0,12$ & $-0,95$ & & $3,99 \pm 1,12$ & 1,00 & 5,00 & $-0,84$ & $-0,23$ \\
\hline Item 18 & $2,53 \pm 1,34$ & 1,00 & 5,00 & 0,40 & $-1,04$ & & $3,29 \pm 1,47$ & 1,00 & 5,00 & $-0,28$ & $-1,29$ \\
\hline Item 19 & $2,59 \pm 1,25$ & 1,00 & 5,00 & 0,31 & $-0,90$ & & $2,90 \pm 1,36$ & 1,00 & 5,00 & 0,10 & $-1,16$ \\
\hline Item 23 & $1,99 \pm 1,21$ & 1,00 & 5,00 & 1,04 & $-0,04$ & & $1,99 \pm 1,27$ & 1,00 & 5,00 & 1,05 & $-0,11$ \\
\hline Item 31 & $2,49 \pm 1,29$ & 1,00 & 5,00 & 0,34 & $-1,02$ & & $2,65 \pm 1,37$ & 1,00 & 5,00 & 0,25 & $-1,16$ \\
\hline $\mathrm{TC}(\alpha=0,66)$ & & & & & & $\mathrm{TC}(\alpha=0,59)$ & & & & & \\
\hline Item 2 & $2,70 \pm 1,31$ & 1,00 & 5,00 & 0,13 & $-1,19$ & & $2,39 \pm 1,34$ & 1,00 & 5,00 & 0,51 & $-0,99$ \\
\hline Item 7 & $2,20 \pm 1,28$ & 1,00 & 5,00 & 0,81 & $-0,48$ & & $2,52 \pm 1,40$ & 1,00 & 5,00 & 0,40 & $-1,13$ \\
\hline Item 12 & $2,64 \pm 1,25$ & 1,00 & 5,00 & 0,30 & $-0,86$ & & $2,64 \pm 1,30$ & 1,00 & 5,00 & 0,31 & $-0,93$ \\
\hline Item 16 & $2,23 \pm 1,18$ & 1,00 & 5,00 & 0,64 & $-0,55$ & & $2,58 \pm 1,31$ & 1,00 & 5,00 & 0,36 & $-1,01$ \\
\hline Item 22 & $2,69 \pm 1,33$ & 1,00 & 5,00 & 0,20 & $-1,13$ & & $3,32 \pm 1,46$ & 1,00 & 5,00 & $-0,33$ & $-1,25$ \\
\hline
\end{tabular}


Oliveira, H. Z.; Dias, T. S.; Sabino, B.; Dias, C.; Corte-Real, N.; Maia, J. A.; Fonseca, A.

\begin{tabular}{lllllllllll} 
Item 24 & $2,68 \pm 1,33$ & 1,00 & 5,00 & 0,19 & $-1,16$ & $2,89 \pm 1,53$ & 1,00 & 5,00 & 0,09 & $-1,45$ \\
Item 27 & $3,00 \pm 1,25$ & 1,00 & 5,00 & $-0,10$ & $-0,95$ & $2,98 \pm 1,33$ & 1,00 & 5,00 & $-0,02$ & $-1,09$ \\
Item 29 & $2,84 \pm 1,37$ & 1,00 & 5,00 & 0,05 & $-1,23$ & $2,67 \pm 1,44$ & 1,00 & 5,00 & 0,30 & $-1,23$ \\
\hline
\end{tabular}

$\mathrm{ET}=$ Envolvimento para a Tarefa; $\mathrm{SA}=$ Suporte à Autonomia; $\mathrm{SS}=$ Suporte Social; EE= Envolvimento ao Ego; $\mathrm{TC}=$ Treino Controlador.

Os valores das estimativas das cargas fatoriais não estandardizadas e as variâncias-erro entre os atletas dos dois países estão na tabela 2. Os resultados sugerem similaridades entre os atletas portugueses e brasileiros.

Tabela 2.

Carga fatorial não estandardizada e variância dos erros.

\begin{tabular}{|c|c|c|c|c|}
\hline & \multicolumn{2}{|c|}{ Portugueses } & \multicolumn{2}{|c|}{ Brasileiros } \\
\hline Item & $\begin{array}{r}\text { Carga } \\
\text { Fatorial } \pm \\
\text { Erro Padrão }\end{array}$ & $\begin{array}{r}\text { Variância- } \\
\text { Erro }\end{array}$ & $\begin{array}{r}\text { Carga } \\
\text { Fatorial } \pm \\
\text { Erro Padrão }\end{array}$ & $\begin{array}{r}\text { Variância- } \\
\text { Erro }\end{array}$ \\
\hline \multicolumn{5}{|l|}{ ET } \\
\hline Item 1 & 1,00 & $0,29 \pm 0,02$ & 1,00 & $0,48 \pm 0,03$ \\
\hline Item 4 & $1,13 \pm 0,08$ & $0,28 \pm 0,02$ & $1,03 \pm 0,10$ & $0,53 \pm 0,03$ \\
\hline Item 11 & $1,02 \pm 0,08$ & $0,38 \pm 0,03$ & $1,02 \pm 0,10$ & $0,57 \pm 0,04$ \\
\hline Item 13 & $0,53 \pm 0,08$ & $0,41 \pm 0,04$ & $0,74 \pm 0,09$ & $0,49 \pm 0,03$ \\
\hline Item 17 & $0,90 \pm 0,08$ & $0,44 \pm 0,03$ & $1,51 \pm 0,12$ & $0,43 \pm 0,03$ \\
\hline Item 21 & $1,11 \pm 0,09$ & $0,44 \pm 0,03$ & $1,54 \pm 0,13$ & $0,55 \pm 0,04$ \\
\hline Item 26 & $1,16 \pm 0,09$ & $0,44 \pm 0,03$ & $1,34 \pm 0,12$ & $0,52 \pm 0,04$ \\
\hline Item 28 & $1,07 \pm 0,09$ & $0,44 \pm 0,03$ & $1,25 \pm 0,11$ & $0,56 \pm 0,04$ \\
\hline Item 32 & $0,81 \pm 0,06$ & $0,20 \pm 0,02$ & $0,75 \pm 0,08$ & $0,35 \pm 0,02$ \\
\hline \multicolumn{5}{|l|}{ SA } \\
\hline Item 3 & 1,00 & $0,88 \pm 0,07$ & 1,00 & $0,89 \pm 0,06$ \\
\hline Item 6 & $0,65 \pm 0,13$ & $0,63 \pm 0,05$ & $0,28 \pm 0,08$ & $0,94 \pm 0,06$ \\
\hline Item 15 & $1,37 \pm 0,19$ & $0,43 \pm 0,04$ & $1,19 \pm 0,11$ & $0,83 \pm 0,06$ \\
\hline Item 20 & $1,05 \pm 0,16$ & $0,56 \pm 0,04$ & $0,76 \pm 0,09$ & $0,85 \pm 0,06$ \\
\hline Item 30 & $0,73 \pm 0,12$ & $0,43 \pm 0,03$ & $0,36 \pm 0,07$ & $0,79 \pm 0,05$ \\
\hline \multicolumn{5}{|l|}{ SS } \\
\hline Item 8 & 1,00 & $0,38 \pm 0,04$ & 1,00 & $0,63 \pm 0,05$ \\
\hline Item 14 & $0,63 \pm 0,09$ & $1,13 \pm 0,08$ & $0,61 \pm 0,08$ & $1,15 \pm 0,07$ \\
\hline Item 25 & $0,81 \pm 0,09$ & $1,02 \pm 0,08$ & $0,96 \pm 0,09$ & $1,20 \pm 0,08$ \\
\hline \multicolumn{5}{|l|}{$\mathbf{E E}$} \\
\hline Item 5 & 1,00 & $1,41 \pm 0,10$ & 1,00 & $1,51 \pm 0,09$ \\
\hline Item 9 & $1,75 \pm 0,28$ & $1,02 \pm 0,08$ & $2,52 \pm 0,43$ & $1,21 \pm 0,09$ \\
\hline Item 10 & $1,00 \pm 0,20$ & $1,38 \pm 0,10$ & $0,66 \pm 0,17$ & $1,16 \pm 0,07$ \\
\hline Item 18 & $1,79 \pm 0,29$ & $1,14 \pm 0,09$ & $2,52 \pm 0,43$ & $1,16 \pm 0,09$ \\
\hline Item 19 & $1,45 \pm 0,24$ & $1,12 \pm 0,08$ & $1,95 \pm 0,35$ & $1,34 \pm 0,08$ \\
\hline
\end{tabular}

\begin{tabular}{lrrrr} 
Item 23 & $1,84 \pm 0,28$ & $0,74 \pm 0,06$ & $2,15 \pm 0,37$ & $0,98 \pm 0,07$ \\
Item 31 & $1,83 \pm 0,29$ & $0,97 \pm 0,08$ & $2,23 \pm 0,38$ & $1,17 \pm 0,08$ \\
TC & & & & \\
Item 2 & 1,00 & $1,39 \pm 0,10$ & 1,00 & $1,37 \pm 0,09$ \\
Item 7 & $1,49 \pm 0,20$ & $0,93 \pm 0,08$ & $0,90 \pm 0,12$ & $1,61 \pm 0,10$ \\
Item 12 & $1,40 \pm 0,19$ & $0,95 \pm 0,08$ & $1,17 \pm 0,13$ & $1,09 \pm 0,08$ \\
Item 16 & $1,63 \pm 0,21$ & $0,57 \pm 0,06$ & $1,38 \pm 0,14$ & $0,89 \pm 0,07$ \\
Item 22 & $0,80 \pm 0,15$ & $1,60 \pm 0,11$ & $0,85 \pm 0,12$ & $1,79 \pm 0,11$ \\
Item 24 & $0,98 \pm 0,16$ & $1,47 \pm 0,11$ & $0,96 \pm 0,13$ & $1,94 \pm 0,12$ \\
Item 27 & $0,30 \pm 0,13$ & $1,53 \pm 0,11$ & $-0,17 \pm 0,10$ & $1,75 \pm 0,11$ \\
Item 29 & $0,21 \pm 0,14$ & $1,85 \pm 0,13$ & $0,23 \pm 0,11$ & $2,05 \pm 0,12$ \\
\hline
\end{tabular}

$\mathrm{ET}=$ Envolvimento para a Tarefa; $\mathrm{SA}=$ Suporte à Autonomia; $\mathrm{SS}=$ Suporte Social; $\mathrm{EE}=$ Envolvimento ao Ego; $\mathrm{TC}=$ Treino Controlador.

As matrizes de variância e covariância entre os fatores de primeira ordem estão na tabela 3, sugerindo um padrão de similaridade entre os atletas dos dois países. Entretanto os resultados evidenciaram valores abaixo do adotado na metodologia para a validade convergente e discriminante. Importa ressaltar também que o padrão das covariâncias e das correlações entre os fatores sugerem uma associação dos 5 fatores num agrupamento superior de segunda ordem Envolvimento para a Tarefa, Suporte à Autonomia e Suporte Social; Envolvimento ao Ego e Treino Controlador.

Os valores dos índices de ajustamento global são apresentados na tabela 4 através da AFC. O modelo 1 contemplou uma estrutura de primeira ordem sem restrições. $\mathrm{O}$ modelo 2 foi definido com base nas sugestões de modificações (erros de medida correlacionados: e6-e30; e9-e18; e10e22; e11-e13; e21-e26; e27-29). O modelo 3 contemplou uma estrutura de segunda ordem sem restrições e o modelo 4 foi definido com uma estrutura de segunda ordem e os mesmos erros correlacionados do modelo 2 . 


\section{Adaptação transcultural do Empowering Disempowering}

Tabela 3.

Fiabilidade Compósita (FC), validade convergente, discriminante, matrizes de variâncias e Covariâncias (superior) e correlação ao quadrado entre os fatores de primeira ordem (inferior).

\begin{tabular}{|c|c|c|c|c|c|c|c|c|c|c|c|c|c|c|c|}
\hline & \multicolumn{7}{|c|}{ Portugueses } & & \multicolumn{7}{|c|}{ Brasileiros } \\
\hline & $\mathrm{FC}$ & VEM & 1 & 2 & 3 & 4 & 5 & & $\mathrm{FC}$ & VEM & 1 & 2 & 3 & 4 & 5 \\
\hline 1-ET & 0,86 & 0,42 & $\mathbf{0 , 3 0}$ & 0,23 & 0,37 & $-0,16$ & $-0,16$ & 1-ET & 0,83 & 0,36 & 0,24 & 0,27 & 0,30 & $-0,12$ & $-0,18$ \\
\hline 2-AS & 0,59 & 0,23 & 0,88 & 0,22 & 0,30 & $-0,10$ & $-0,11$ & 2-SA & 0,51 & 0,20 & 0,79 & 0,40 & 0,44 & $-0,16$ & $-0,26$ \\
\hline 3-SS & 0,56 & 0,32 & 0,92 & 0,83 & $\mathbf{0 , 5 0}$ & $-0,21$ & $-0,24$ & 3-SS & 0,53 & 0,28 & 0,74 & 0,94 & 0,51 & $-0,17$ & $-0,30$ \\
\hline 4-EE & 0,74 & 0,31 & 0,41 & 0,25 & 0,44 & 0,21 & 0,22 & 4-EE & 0,71 & 0,28 & 0,44 & 0,52 & 0,44 & 0,14 & 0,20 \\
\hline 5-TC & 0,65 & 0,24 & 0,30 & 0,24 & 0,41 & 0,79 & $\mathbf{0 , 3 1}$ & 5-TC & 0,59 & 0,20 & 0,35 & 0,41 & 0,42 & 0,72 & 0,44 \\
\hline
\end{tabular}

$\mathrm{ET}=$ Envolvimento para a Tarefa; $\mathrm{SA}=$ Suporte à Autonomia; $\mathrm{SS}=$ Suporte Social; $\mathrm{EE}=\mathrm{Envolvimento}$ ao Ego; $\mathrm{TC}=\mathrm{Treino}$

Controlador.

Tabela 4.

Índices globais de ajustamento

\begin{tabular}{|c|c|c|c|c|c|c|c|c|c|c|c|c|}
\hline & \multicolumn{6}{|c|}{ Portugueses } & \multicolumn{6}{|c|}{ Brasileiros } \\
\hline & $\chi^{2} / \mathrm{df}$ & CFI & TLI & SRMR & RMSEA & RMSEA 90\% IC & $\chi^{2} / \mathrm{df}$ & $\mathrm{CFI}$ & TLI & SRMR & RMSEA & RMSEA $90 \%$ IC \\
\hline $\begin{array}{l}\text { Modelo } 1 \\
\text { ( } 1^{\mathrm{a} o r d e m}, \text { sem } \\
\text { restrições })\end{array}$ & 2,16 & 0,86 & 0,85 & 0,06 & 0,05 & $0,05-0,06$ & 2,28 & 0,86 & 0,85 & 0,06 & 0,05 & $0,04-0,05$ \\
\hline $\begin{array}{l}\text { Modelo } 2\left(1^{\text {a }}\right. \\
\text { ordem, } \\
\text { correlação entre } \\
\text { erros de } \\
\text { medidas* })\end{array}$ & 1,66 & 0,92 & 0,90 & 0,06 & 0,04 & $0,04-0,05$ & 1,93 & 0,90 & 0,89 & 0,05 & 0,04 & $0,04-0,05$ \\
\hline $\begin{array}{l}\text { Modelo } 3\left(2^{\mathrm{a}}\right. \\
\text { ordem, sem } \\
\text { restrições) }\end{array}$ & 2,17 & 0,86 & 0,85 & 0,06 & 0,05 & $0,05-0,06$ & 2,26 & 0,86 & 0,85 & 0,06 & 0,05 & $0,04-0,05$ \\
\hline $\begin{array}{l}\text { Modelo } 4\left(2^{\mathrm{a}}\right. \\
\text { ordem, } \\
\text { correlação entre } \\
\text { erros de } \\
\text { medidas*) }\end{array}$ & 1,67 & 0,92 & 0,91 & 0,06 & 0,04 & $0,04-0,05$ & 1,92 & 0,90 & 0,89 & 0,05 & 0,04 & $0,04-0,05$ \\
\hline
\end{tabular}

*Correlação entre os erros (e6-e30; e9-e18; e10-e22; e11-e13; e21-e26; e27-29).

A partir do modelo 4, nas figuras 1 e 2 estão representados os pesos fatoriais ajustados $\mathrm{e}$ as correlações para os modelos de segunda ordem com atletas portugueses e brasileiros. 
Oliveira, H. Z.; Dias, T. S.; Sabino, B.; Dias, C.; Corte-Real, N.; Maia, J. A.; Fonseca, A.

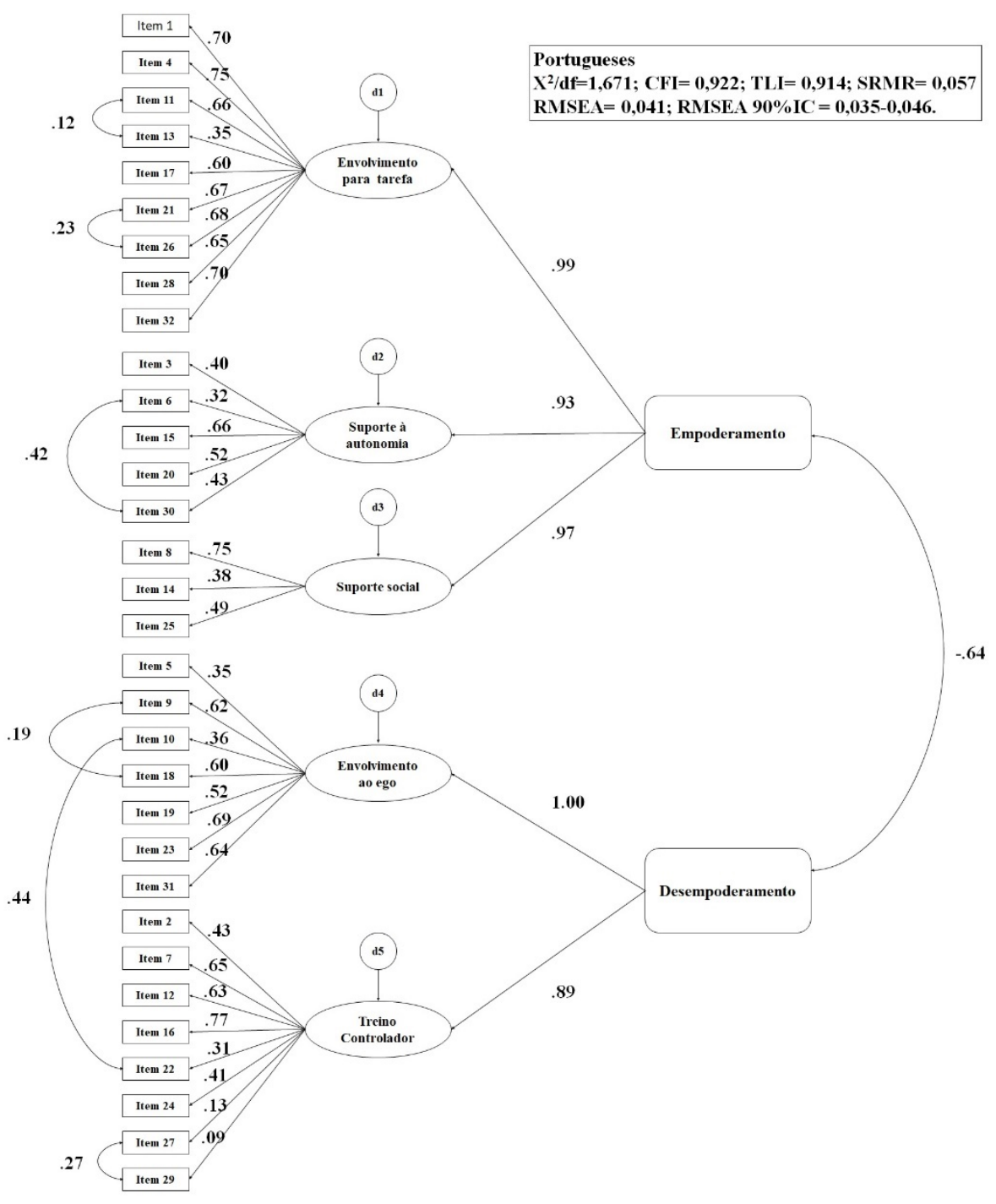

Figura 1. Pesos fatoriais ajustados e correlações entre fatores para o modelo com atletas portugueses. 


\section{Adaptação transcultural do Empowering Disempowering}

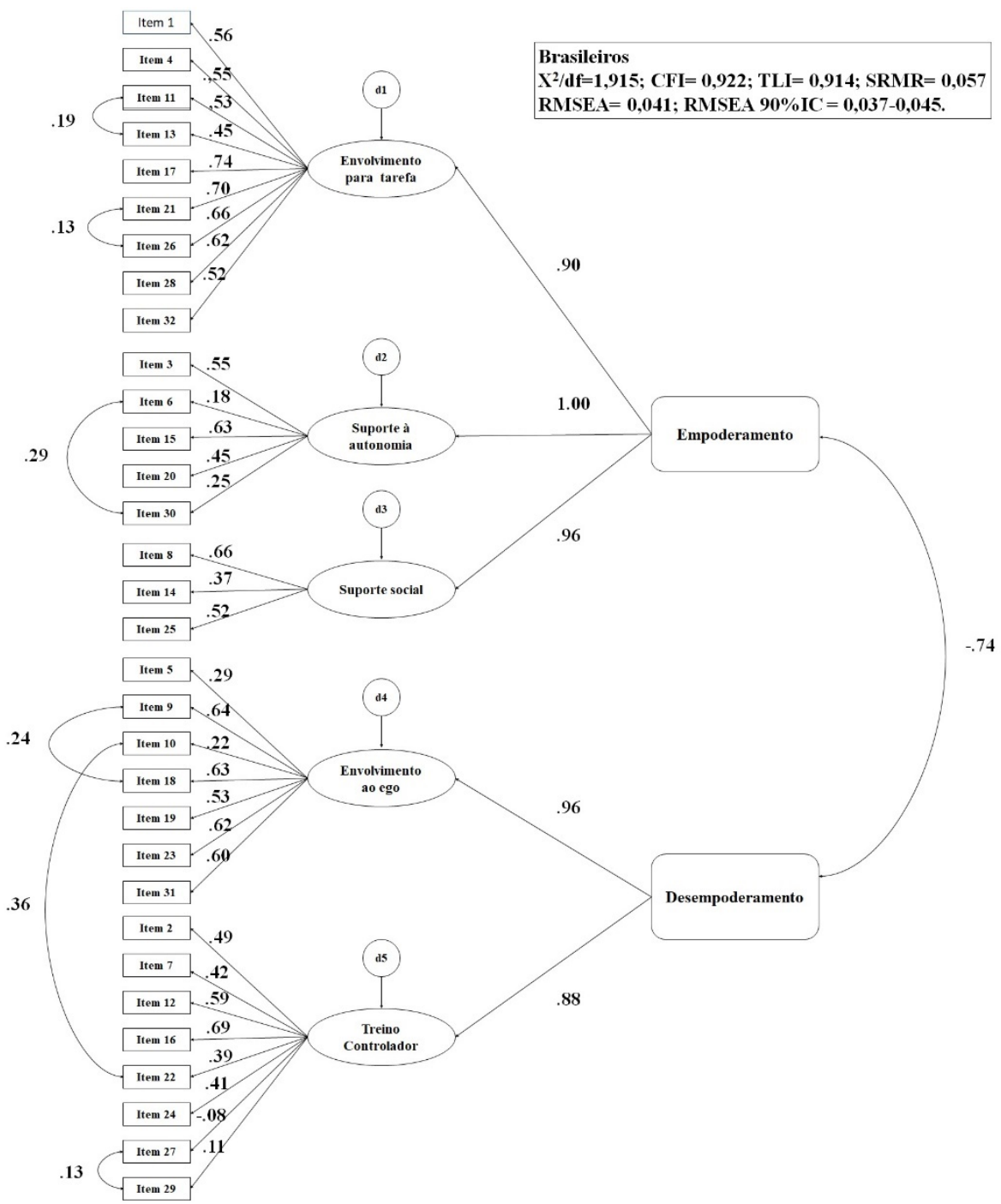

Figura 2. Pesos fatoriais ajustados e correlações entre fatores para o modelo com atletas brasileiros.

Os resultados das análises da invariância do modelo de segunda ordem entre os atletas portugueses e brasileiros são apresentados na tabela 5, através da qual é possível confirmar a $\begin{array}{r}\text { invariância } \\ \text { configuracional, de medida }\end{array}$ e escalar. 
Oliveira, H. Z.; Dias, T. S.; Sabino, B.; Dias, C.; Corte-Real, N.; Maia, J. A.; Fonseca, A.

Tabela 5.

Invariância dos modelos para os atletas portugueses e brasileiros

\begin{tabular}{|c|c|c|c|c|c|c|}
\hline Modelos testados & $\chi^{2}(\mathrm{df})$ & TLI & SRMR & RMSEA & CFI & $\Delta \mathrm{CFI}$ \\
\hline $\begin{array}{l}\text { Invariância configuracional - Modelo sem } \\
\text { restrições, }\end{array}$ & $1620,86(904)$ & ,90 & 0,57 & 0,03 & 0,91 & - \\
\hline $\begin{array}{l}\text { Invariância de medida I - Cargas fatoriais } \\
\text { de primeira ordem fixas, }\end{array}$ & $1723,07(931)$ & ,90 & 0,61 & 0,03 & 0,90 & 0,01 \\
\hline $\begin{array}{l}\text { Invariância de medida II - Cargas fatoriais } \\
\text { de primeira e segunda ordem fixas, }\end{array}$ & $1735,01(934)$ &, 89 & 0,61 & 0,03 & 0,90 & 0,01 \\
\hline $\begin{array}{l}\text { Invariância escalar I - Cargas fatoriais de } \\
\text { primeira e segunda ordem fixas; interceptos } \\
\text { de primeira ordem fixos, }\end{array}$ & $1737,96(937)$ &, 89 & 0,63 & 0,03 & 0,90 & 0,01 \\
\hline $\begin{array}{l}\text { Invariância escalar II - Cargas fatoriais de } \\
\text { primeira e segunda ordem fixas; interceptos } \\
\text { de primeira e segunda ordem fixos. }\end{array}$ & $1741,50(942)$ & ,90 & 0,63 & 0,03 & 0,90 & 0,01 \\
\hline
\end{tabular}

\section{DISCUSSÃO}

O presente estudo teve como objetivo realizar a tradução e adaptação transcultural para a língua portuguesa do EDMCQ-C e investigar a sua invariância fatorial entre atletas brasileiros $\mathrm{e}$ portugueses. De um modo geral, os resultados das análises desenvolvidas ao longo do estudo refletiram uma estrutura com 32 itens, 5 fatores de primeira ordem e 2 fatores de segunda ordem para atletas dos dois países. Ficou demonstrada também as invariâncias configuracional, escalar e de medida entre os atletas portugueses e brasileiros.

Todavia, os resultados também evidenciaram alguns problemas na solução proposta para os atletas de ambos os países. Relativamente à fiabilidade dos dados, os valores de $\alpha$ dos fatores Envolvimento para a Tarefa e Envolvimento ao Ego, correspondente à TOR foram adequados $\left(\alpha_{\mathrm{ETpt}}=.86 ; \alpha_{\mathrm{ETbr}}=.84 ; \alpha_{\mathrm{EEpt}}\right.$ $\left.=.74 ; \alpha_{\text {EEbr }}=.72\right)$. Em contrapartida, os valores de $\alpha$ para os outros fatores de primeira ordem, apesar de estarem abaixo do recomendado na literatura, ficaram próximos ou foram superiores do reportado no artigo original (Appleton et al., 2016). Sobre a fiabilidade de variáveis latentes que analisam o clima motivacional, Newton et al. (2000) também reportaram valores de $\alpha$ abaixo do esperado na validação do Perceived Motivational Climate Sport Questionnaire-2 (PMCSQ-2). Assim, os resultados do presente estudo foram similares aos de Appleton et al., (2016) e Newton et al. (2000). Importa referir que nesses estudos os menores valores de $\alpha$ estiveram associados aos fatores com menor número de itens dos fatores. Outras possíveis causas dos baixos valores de $\alpha$ podem estar associadas a pouca variância nas respostas dos itens e ao tamanho amostral (Cronbach, 1951; Kline, 2012).

Sobre os resultados referente aos pesos fatoriais, alguns itens apresentaram baixos valores de saturação aos fatores propostos pelo modelo teórico. Por exemplo as cargas fatoriais dos itens 27 e 29 foram as que apresentaram os menores valores nos modelos propostos com atletas dos dois países, de maneira semelhante, os itens 6 e 30 apresentaram baixas cargas fatoriais para atletas brasileiros, o que em alguma medida corrobora com o estudo original do EDMCQ-C por apresentar os mesmos itens com baixas saturações principalmente nos fatores Treino Controlador e Suporte à Autonomia. Devido à similaridade dos resultados do presente estudo com o original no que se refere aos pesos fatoriais dos itens, optou-se pela manutenção da estrutura do modelo.

Os resultados das correlações entre os fatores de primeira ordem, os valores da variância extraída média e dos pesos fatoriais evidenciaram problemas de validade discriminante e convergente do construto. Outros estudos de natureza quantitativa sobre o clima motivacional no ambiente desportivo também reportaram correlações elevadas e problemas similares ao presente estudo (e.g., Reinboth et al., 2004; Appleton et al., 2016). Sobre esses resultados, Appleton et al. (2016), no estudo original do EDMCQ-C, sugeriram a existência de uma sobreposição semântica dos itens, causada pela origem de conceitos de diferentes teorias. Não 


\section{Adaptação transcultural do Empowering Disempowering}

obstante, Appleton et al. (2016) defendem a existência de todos os fatores como previsto por Duda (2013). Além disso, Chen, Sousa e West (2005) afirmaram que altas correlações entre os fatores de primeira ordem, sugerem a existência de fatores de segunda ordem em acordo com os pressupostos teóricos de Duda (2013), os achados de Appleton (2016) e os resultados do presente estudo.

Em relação aos índices globais de ajustamento dos modelos verificou-se índices aceitáveis para os modelos com erros de medidas de alguns itens correlacionados. Esse procedimento tem sido aceito na literatura desde que isso não coloque em causa a identificação do modelo, como no presente estudo (Byrne, 2010). Além disso, os índices de ajustamento associado a natureza correlacional do modelo de primeira ordem, demonstrou-se plausível a adição dos fatores de segunda ordem para a amostra estudada com portugueses e brasileiros, em acordo com o escopo teórico proposto por Duda (2013) e encontrado por Appleton et al., (2016) com atletas do Reino Unido.

No que se refere a invariância do modelo entre a amostra de brasileiros e portugueses, verificou-se uma equivalência na estrutura fatorial nos modelos de primeira e segunda ordem, ou seja, os itens utilizados para explicar os fatores do instrumento não diferem estruturalmente nos atletas dos dois países nos fatores. Desta forma, o modelo de medida proposto pode ser usado nos dois países da mesma maneira.

Ao considerar a invariância do modelo de medida apresentado, principalmente no que concerne aos resultados do $\triangle C F I$, verifica-se que os pesos fatoriais dos itens são equivalentes, sendo possível afirmar que que os itens possuem o mesmo poder explicativo nos dois grupos (Byrne, 2010). Além disso, a invariância escalar também foi demonstrada no presente estudo, permitindo a realização de comparações entre as perceções dos atletas do sexo masculino dos dois países para o clima motivacional. Em suma, o presente estudo verificou a equivalência semântica e operacional do presente instrumento, com base na TOR e na TAD, para atletas brasileiros e portugueses.

A invariância dos instrumentos de avaliação psicológica entre pessoas de diferentes países tem sido analisada na literatura em diferentes contextos como trabalho (e.g., Brien et al., 2012), educacional (e.g., Wood et al., 2012) e desportivo (e.g., González, Balaguer, Corte-Real, \& Fonseca, 2016). Porém a comparação de modelos em jovens brasileiros e portugueses parece ser menos frequente e mais associado ao contexto educacional. Nesse sentido, curioso notar que os resultados entre os dois países lusófonos no contexto educacional não corroboram com os resultados do presente estudo por não conseguir demonstrar a invariância escalar (Campos, Carlotto, \& Marôco, 2013; Campos \& Marôco, 2012; Marôco, Campos, Vinagre, \& Pais-Ribeiro, 2014; Marôco, Campos, Bonafé, Vinagre, \& Pais-Ribeiro, 2014). No contexto desportivo, as pesquisas que analisaram a invariância entre brasileiros e portugueses são ainda mais escassas. Entretanto, com base na TAD, ficou comprovada a invariância das necessidades psicológicas básicas entre estudantes para as aulas de educação física nos dois países (Cid et al., 2016). De uma maneira geral, ainda que reduzido os estudos que testaram a invariância entre brasileiros e portugueses, as pesquisas demonstram não ser frequente a invariância, como encontrada no presente estudo.

Por fim, de uma maneira geral os resultados do presente estudo evidenciam uma estrutura do clima motivacional multidimensional e hierárquico tanto para atletas brasileiros e quanto para portugueses do sexo masculino praticantes de desportos coletivos, em acordo com o modelo teórico proposto por Duda (2013) e empiricamente demonstrado por Appleton et al. (2016). De posse desse instrumento novas pesquisas poderão ser desenvolvidas com amostras mais heterogêneas, em estudos longitudinais e associado as consequências dos climas motivacionais percebido.

Quanto as limitações do estudo, importa referir que alguns dos resultados indicaram problemas na construção dos itens como reportado no instrumento original. Adicionalmente $\mathrm{o}$ presente estudo não considerou diferentes formas de validade (e.g. validade preditiva; validade cruzada). Outra limitação, refere-se à natureza multinível dos dados, uma vez que a perceção dos atletas está condicionada aos treinadores, que por sua vez sofrem interferência dos clubes, assim outras estratégias de análise que consideram as características multinível dos dados 
Oliveira, H. Z.; Dias, T. S.; Sabino, B.; Dias, C.; Corte-Real, N.; Maia, J. A.; Fonseca, A.

poderão ser aplicadas (Appleton et al., 2016; Smith et al., 2016).

\section{IMPLICAÇÕES PRÁTICAS}

Em conclusão, os resultados do presente estudo, desenvolvido sob uma perspectiva de natureza confirmatória, permite assumir que estão reunidas condições para considerar como válida a versão brasileira e portuguesa do EDMCQ-C para avaliar a perceção dos atletas sobre o clima motivacional proporcionado pelo treinador. Adotar a conceituação teórica integrada proposta por Duda (2013) é vantajosa na medida em que amplia as dimensões do clima motivacional centrais na TOR e na TAD. Assim, esse instrumento poderá ser útil a treinadores e psicólogos desportivos que buscam analisar o clima motivacional de suas equipes. Poderá também ser útil a pesquisadores que busquem compreender as associações e as consequências do clima motivacional ligadas a prática desportiva dos atletas, possibilitando um maior aprofundamento do conhecimento disponível nesse âmbito.

\section{REFERENCIAS}

1. Ames, C. (1992). Achievement goals and adaptative motivational patterns: The role of the environment. In G. C. Roberts (Ed.), Motivation in Sport and Exercise (pp. 161-176). Champaign, IL: Human Kinetics.

2. Appleton, P. R., \& Duda, J. L. (2016). Examining the interactive effects of coach-created empowering and disempowering climate dimensions on athletes' health and functioning. Psychology of Sport and Exercise, 26, 61-70. DOI:

https://doi.org/10.1016/j.psychsport.2016.06.007

3. Appleton, P. R., Ntoumanis, N., Quested, E., Viladrich, C., \& Duda, J. L. (2016). Initial validation of the coach-created Empowering and Disempowering Motivational Climate Questionnaire (EDMCQ-C). Psychology of Sport and Exercise, 22, 53-65. DOI: https://doi.org/10.1016/j.psychsport.2015.05.008

4. Ato, M., López-García, J. J., \& Benavente, A. (2013). A classification system for research designs in psychology. Annals of Psychology,
29(3), 1038-1059.

http://dx.doi.org/10.6018/analesps.29.3.178511

5. Bartholomew, K. J., Ntoumanis, N., \& Thogersen-Ntoumani, C. (2009). A review of controlling motivational strategies from a selfdetermination theory perspective: implications for sports coaches. International Review of Sport and Exercise Psychology, 2(2), 215-233. DOI: https://doi.org/10.1080/17509840903235330

6. Bentler, P. M., \& Bonett, D. G. (1980). Significance tests and goodness of fit in the analysis of covariance structures. Psychological Bulletin, $\quad 88(3), \quad$ 588-606. https://doi.org/10.1037/0033-2909.88.3.588

7. Brien, M., Forest, J., Mageau, G. A., Boudrias, J., Desrumaux, P., Brunet, L., \& Morin, E. M. (2012). The basic psychological needs at work scale: Measurement invariance between Canada and France. Applied Psychology: Health and Well-Being, 4(2), 167-187. DOI: https://doi.org/10.1111/j.1758$\underline{0854.2012 .01067 . x}$

8. Brislin, R. W. (1980). Translation and content analysis of oral and written materials. In $\mathrm{H}$. Triandis \& J. Berry (Eds.), Handbook of CrossCultural Psychology (Vol. 2, pp. 389-444). Needam Heights, MA: Allyn and Bacon.

9. Borrego, C. C., \& Silva, C. (2012). Propriedades psicométricas da versão portuguesa para jovens atletas de basquetebol do Motivational Climate Scale for Youth Sports. Cuadernos de Psicología del Deporte, 12(Supl.1), 5-8. DOI: https://doi.org/10.4321/S157884232012000300002

10. Burgueño, R., Granero-Gallegos, A., AlcarazIbáñez, M., Sicilia, A., \& Medina-Casaubón, J. (2018). La necesidad de medir la motivación situacional en el contexto español de la educación física: Psicometría de la Situational Motivation Scale. Cuadernos de psicología del deporte, 18(2), 135-151.

11. Byrne, B. M. (2010). Structural equation modeling with AMOS: Basic concepts, applications, and programming (2nd ed.): Routledge.

12. Byrne, B. M., \& Stewart, S. M. (2006). The MACS approach to testing for multigroup 


\section{Adaptação transcultural do Empowering Disempowering}

invariance of a second-order structure: A walk through the process. Structural equation modeling, 13(2), 287-321. DOI: https://doi.org/10.1207/s15328007sem1302 7

13. Campos, J. A. D. B., Carlotto, M. S., \& Marôco, J. (2013). Copenhagen Burnout Inventory-student version: adaptation and transcultural validation for Portugal and Brazil. Psicologia: Reflexão e Crítica, 26(1), 87-97. DOI: http://dx.doi.org/10.1590/S010279722013000100010

14. Campos, J. A. D. B., \& Marôco, J. (2012). Adaptação transcultural Portugal-Brasil do Inventário de Burnout de Maslach para estudantes. Revista de Saúde Pública, 46, 816824. DOI: http://dx.doi.org/10.1590/S003489102012000500008

15. Cantú-Berrueto, A., Castillo, I., López-Walle, J., Tristán, J., \& Balaguer, I. (2016). Estilo interpersonal del entrenador, necesidades psicológicas básicas y motivación: Un estudio en futbolistas universitarios mexicanos. Revista Iberoamericana de Psicología Del Ejercicio y El Deporte, $\quad 11(2), \quad 263-270 . \quad$ DOI: https://doi.org/10.5093/rpadef2017a2

16. Castillo, I., Duda, J. L., Álvarez, M. S., Mercé, J., \& Balaguer, I. (2010). Clima motivacional, metas de logro de aproximación y evitación y bienestar en futbolistas cadetes. Revista de Psicología del Deporte, 20(1), 149-164.

17. Castillo, I., Ramis, Y., Cruz, J., \& Balaguer, I. (2015). Formación de entrenadores de fútbol de base em el proyecto PAPA. Revista de Psicología del Deporte, 24 (1), 131-138.

18. Chen, F. F., Sousa, K. H., \& West, S. G. (2005). Teacher's corner: Testing measurement invariance of second-order factor models. Structural equation modeling, 12(3), 471-492. DOI: https://doi.org/10.1207/s15328007sem1203 7

19. Cheung, G. W., \& Rensvold, R. B. (2002). Evaluating Goodness-of-Fit Indexes for Testing Measurement Invariance. Structural Equation Modeling: A Multidisciplinary Journal, 9(2), 233-255.

DOI: https://doi.org/10.1207/S15328007SEM0902 5
20. Cid, L., Lettnin, C., Stobäus, C., Monteiro, D., Davoglio, T., \& Moutão, J. M. (2016). Crosscultural validation of the basic psychological needs in Physical Education Scale between Portugal and Brazil samples. The Spanish journal of psychology, 19(e5),1-10. DOI: https://doi.org/10.1017/sjp.2016.6

21. Cronbach, L. J. (1951). Coefficient alpha and the internal structure of tests. Psychometrika, 16(3), 297-334.

DOI: http://dx.doi.org/10.1007/BF02310555

22. Deci, E. L., \& Ryan, R. M. (1985). Intrinsic motivation and self-determination in human behavior. New York: Plenum. DOI: https://doi.org/10.1007/978-1-4899-2271-7

23. Deci, E. L., \& Ryan, R. M. (2000). The" what" and" why" of goal pursuits: Human needs and the self-determination of behavior. Psychological Inquiry, 11(4), 227-268. DOI: https://doi.org/10.1207/S15327965PLI1104 01

24. DeFreese, J. D., \& Smith, A. L. (2013). Teammate social support, burnout, and selfdetermined motivation in collegiate athletes. Psychology of Sport and Exercise, 14(2), 258265.

DOI: https://doi.org/10.1016/j.psychsport.2012.10.009

25. Dias, T. S., Novotná, K., Oliveira, H. Z., Azevedo, C., Corte-Real, N., Slepička, P., \& Fonseca, A. M. (2018). Why talented athletes drop out from sport? The Portuguese and Czech case. Education + Training, 60(5). DOI: https://doi.org/10.1108/ET-12-2017-0207

26. Duarte, D., Garganta, J., \& Fonseca, A. M. (2012). Adaptação transcultural e avaliação das propriedades psicométricas e estrutura factorial da versão portuguesa da Coaching Efficacy Scale (CESp). Revista Portuguesa de Ciências do Desporto, 12(3). DOI: https://doi.org/10.5628/rpcd.12.03.12

27. Duda, J. L. (2013). The conceptual and empirical foundations of Empowering Coaching ${ }^{\mathrm{TM}}$ : Setting the stage for the PAPA project. International Journal of Sport and Exercise Psychology, 11(4), 311-318.

DOI: https://doi.org/10.1080/1612197X.2013.839414

28. Duda, J. L., \& Balaguer, I. (2007). Coach-created motivational climate. In S. Jowett \& D. Lavallee 
Oliveira, H. Z.; Dias, T. S.; Sabino, B.; Dias, C.; Corte-Real, N.; Maia, J. A.; Fonseca, A.

(Eds.), Social Psychology in Sport (pp. 117-130): Champaing,IL:Human Kinetics.

29. Duda, J. L., Quested, E., Haug, E., Samdal, O., Wold, B., Balaguer, I., Castillo, I., Sarrazin, P., Papaioannou, A., \& Ronglan, L. T. (2013). Promoting Adolescent health through an intervention aimed at improving the quality of their participation in Physical Activity (PAPA): Background to the project and main trial protocol. International Journal of Sport and Exercise Psychology, 11(4), 319-327. DOI: https://doi.org/10.1080/1612197X.2013.839413

30. Fenton, S. A. M., Duda, J. L., Appleton, P. R., \& Barrett, T. G. (2017). Empowering youth sport environments: Implications for daily moderateto-vigorous physical activity and adiposity. Journal of Sport and Health Science, 6, 423-433. DOI: https://doi.org/10.1016/j.jshs.2016.03.006

31. Figueiras, T., Santana, P., Corte-Real, N., Dias, C., Brustad, R., \& Fonseca, A. M. (2010). Análise da estrutura factorial e da invariância da versão portuguesa da Satisfaction with Life Scale (SWLSp) quando aplicada a adultos de ambos os sexos. Revista Portuguesa de Ciências do Desporto, $\quad 10(3), \quad 11-30 . \quad$ DOI: https://doi.org/10.5628/rpcd.10.03.11

32. Finney, S. J., \& DiStefano, C. (2006). Nonnormal and categorical data in structural equation modeling. In G. R. Hacock \& R. O. Mueller (Eds.), Structural equation modeling: A second course (Vol. 10, pp. 269-314). Conn: Greenwich.

33. Fonseca, A. M., \& Biddle, S. (1995). Versão portuguesa do Perceived Motivational Climate in Sport Questionnaire. Manuscrito não publicado. FCDEF-UP, Porto, Portugal.

34. Fonseca, A. M., \& Brito, A. P. (2001). Propriedades psicométricas da versão portuguesa do Intrinsic Motivation Inventory (IMIp) em contextos de actividade física e desportiva. Análise Psicológica, 19(1), 59-76. DOI: https://doi.org/10.14417/ap.344

35. Fonseca, A. M., \& Fox, K. (2002). Como avaliar o modo como as pessoas se percebem fisicamente? Um olhar sobre a versão portuguesa do Physical Self-Perception Profile (PSPP). Revista Portuguesa de Ciências do Desporto,
2(5),

11-23.

https://doi.org/10.5628/rpcd.02.05.11

36. González, F. L. A., Balaguer, I., Corte-Real, N., \& Fonseca, A. M. (2016). Factorial invariance of the Satisfaction with Life Scale in adolescents from Spain and Portugal. Psicothema, 28(3), 353-358. DOI: $10.7334 /$ psicothema2016.1

37. Hagger, M. S., \& Chatzisarantis, N. L. D. (2007). Intrinsic motivation and self-determination in exercise and sport: Human Kinetics.

38. Hair, J. F., Anderson, R. E., Tatham, R. L., \& Black, W. C. (2014). Multivariate data analysis (7th ed.). Englewood Cliffs: Prentice Hall.

39. Harwood, C. G., Keegan, R. J., Smith, J. M. J., \& Raine, A. S. (2015). A systematic review of the intrapersonal correlates of motivational climate perceptions in sport and physical activity. Psychology of Sport and Exercise, 18, 9-25. DOI: https://doi.org/10.1016/j.psychsport.2014.11.005

40. Hu, L. t., \& Bentler, P. M. (1999). Cutoff criteria for fit indexes in covariance structure analysis: Conventional criteria versus new alternatives. Structural equation modeling: a multidisciplinary journal, $\quad 6(1), \quad 1-55 . \quad$ DOI: https://doi.org/10.1080/10705519909540118

41. Kline, R. B. (2011). Principles and practice of structural equation modeling. New York: The Guildford Press.

42. Jöreskog, K. G., \& Sörbom, D. (1989). LISREL 7: A guide to the program and applications. Mooresville, IN: Spss.

43. Lemyre, P., Roberts, G. C., \& Stray-Gundersen, J. (2007). Motivation, overtraining, and burnout: Can self-determined motivation predict overtraining and burnout in elite athletes? European Journal of Sport Science, 7(2), 115126.

DOI:

https://doi.org/10.1080/17461390701302607

44. Leo-Marcos, F. M., Sánchez-Miguel, P. A., Sánchez-Oliva, D., Gómez-Corrales, F. R., \& García-Calvo, T. (2009). Análisis de las relaciones existentes entre la orientación y el clima motivacional con los comportamientos antosociales en jóvenes deportistas. Revista Iberoamericana de Psicología Del Ejercicio y El Deporte, 4(1), 15-28. 


\section{Adaptação transcultural do Empowering Disempowering}

45. Mageau, G. A., \& Vallerand, R. J. (2003). The coach-athlete relationship: A motivational model. Journal of Sports Sciences, 21(11), 883904.

DOI: https://doi.org/10.1080/0264041031000140374

46. Marôco, J. (2014). Análise de equações estruturais: Fundamentos teóricos, software \& aplicações. Lisboa: ReportNumber.

47. Marôco, J., Campos, J. A. D. B., Vinagre, M. G., \& Pais-Ribeiro, J. L. (2014). Adaptação transcultural Brasil-Portugal da Escala de Satisfação com o Suporte Social para estudantes do ensino superior. Psicologia: Reflexão e Crítica, 27(2), 247-254. DOI: https://doi.org/10.1590/1678-7153.201427205

48. Marôco, J., Campos, J. B., Bonafé, F. S., Vinagre, M. G., \& Pais-Ribeiro, J. (2014). Adaptação transcultural Brasil-Portugal da escala Brief COPE para estudantes do ensino superior. Psicologia, Saúde e Doenças, 15(2), 300-313. DOI: $10.1590 / 1678-7153.201427205$

49. Monteiro, D., Borrego, C. C., Silva, C., Moutão, J. M., Marinho, D. A., \& Cid, L. (2018). Motivational Climate Sport Youth Scale: Measurement invariance across gender and five different sports. Journal of human kinetics, 61(1), 249-261. DOI: https://doi.org/10.1515/hukin2017-0124

50. Nicholls, J. G. (1984). Achievement motivation: Conceptions of ability, subjective experience, task choice, and performance. Psychological review, 91(3), 328-346. DOI: http://dx.doi.org/10.1037/0033-295X.91.3.328

51. Ntoumanis, N. (2001). Empirical links between achievement goal theory and self-determination theory in sport. Journal of Sports Science, 19(6), 397-409.

DOI: https://doi.org/10.1080/026404101300149357

52. Occhino, J., Mallett, C., Rynne, S., \& Carlisle, K. (2014). Autonomy-supportive pedagogical approach to sports coaching: Research, challenges and opportunities. International Journal of Sports Science and Coaching, 9(2), 401-416. DOI: https://doi.org/10.1260/17479541.9.2.401

53. Pelletier, L. G., Fortier, M. S., Vallerand, R. J., \& Briere, N. M. (2001). Associations among perceived autonomy support, forms of selfregulation, and persistence: A prospective study. Motivation and emotion, 25(4), 279-306. DOI: http://dx.doi.org/10.1023/A:1014805132406

54. Reinboth, M., Duda, J. L., \& Ntoumanis, N. (2004). Dimensions of coaching behavior, need satisfaction, and the psychological and physical welfare of young athletes. Motivation and emotion, 28(3), 297-313. DOI: http://dx.doi.org/10.1023/B:MOEM.0000040156. $\underline{81924 . b 8}$

55. Ryan, R. M., \& Deci, E. L. (2007). Active human nature: Self-determination theory and the promotion and maintenance of sport, exercise, and health. In M. S. Hagger \& N. L. D. Chatzisarantis (Eds.), Intrinsic motivation and self-determination in exercise and sport (pp. 119): Human Kinetics Europe.

56. Ryan, R. M., \& Deci, E. L. (2017). Selfdetermination theory: Basic psychological needs in motivation, development, and wellness. New York: The Guilford Press.

57. Sheridan, D., Coffee, P., \& Lavallee, D. (2014). A systematic review of social support in youth sport. International Review of Sport and Exercise Psychology, 7(1), 198-228. DOI: https://doi.org/10.1080/1750984X.2014.931999

58. Smith, N., Tessier, D., Tzioumakis, Y., Fabra, P., Quested, E., Appleton, P. R., Sarrazin, P., Papaioannou, A., Balaguer, I., \& Duda, J. L. (2016). The relationship between observed and perceived assessments of the coach-created motivational environment and links to athlete motivation. Psychology of Sport and Exercise, 23, 51-63. DOI: https://doi.org/10.1016/j.psychsport.2015.11.001

59. Smith, N., Tessier, D., Tzioumakis, Y., Quested, E., Appleton, P., Sarrazin, P., Papaioannou, A., \& Duda, J. L. (2015). Development and validation of the multidimensional motivational climate observation system. Journal of Sport and Exercise Psychology, 37(1), 4-22. DOI: https://doi.org/10.1123/jsep.2014-0059

60. Spaccarotella, L. (2017). La labor del psicólogo del deporte con la selección Argentina de handball femenino en los Juegos Olímpicos de Brasil 2016. Revista de Psicología Aplicada al 
Oliveira, H. Z.; Dias, T. S.; Sabino, B.; Dias, C.; Corte-Real, N.; Maia, J. A.; Fonseca, A.

Deporte y al Ejercicio Físico, 1(e2), 1-6. DOI: https://doi.org/10.5093/rpadef2017a2

61. Standage, M., Duda, J. L., \& Ntoumanis, N. (2005). A test of self- determination theory in school physical education. British Journal of Educational Psychology, 75(3), 411-433. DOI: https://doi.org/10.1348/000709904X22359

62. Tabachnick, B. G., \& Fidell, L. S. (2007). Using multivariate statistics. Boston, MA: Allyn \& Bacon/Pearson Education.

63. Úbeda-Colomer, J., Pérez-Samaniego, V., \& Devís-Devís, J. (2018). Propiedades psicométricas de un cuestionario de Teoría de la Conducta Planeada en la actividad física en alumnado universitario con discapacidad. Cuadernos de psicología del deporte, 18(2), 317.

64. Wood, G., Pinheiro-Chagas, P., Júlio-Costa, A., Micheli, L. R., Krinzinger, H., Kaufmann, L., . . . Haase, V. G. (2012). Math anxiety questionnaire: similar latent structure in Brazilian and German school children. Child Development Research,12(1),1-10. DOI: http://dx.doi.org/10.1155/2012/610192

65. Vallerand, R. J. (1989). Vers une méthodologie de validation trans-culturelle de questionnaires psychologiques: Implications pour la recherche en langue française. Canadian Psychology/Psychologie Canadienne, 30(4), 662680. 